\title{
Scanning-tunneling-microscopy study on the growth mode of vapor-deposited gold films
}

\author{
P. Herrasti and P. Ocón \\ Departamento de Química Física Aplicada C-II, Universidad Autónoma de Madrid, 28049 Madrid, Spain \\ L. Vázquez \\ Instituto de Ciencia de Materiales, Consejo Superior de Investigaciones Científicas, Departamento Física Aplicada C-XII, \\ Universidad Autónoma de Madrid, 28049 Madrid, Spain \\ R. C. Salvarezza \\ Instituto de Investigaciones Fisicoquímicas Teóricas y Aplicadas, Casilla de Correo 16, Sucursal 4, 1900 La Plata, Argentina \\ J. M. Vara \\ Departamento de Química, Física Aplicada C-II, Universidad Autónoma de Madrid, 28049 Madrid, Spain \\ A. J. Arvia \\ Instituto de Investigaciones Fisicoquímicas Téricas y Aplicadas, Casilla de Correo 16, Sucursal 4, 1900 La Plata, Argentina
}

(Received 18 November 1991)

\begin{abstract}
The growth of gold deposits on smooth glass from the vapor phase at $30 \mathrm{~nm}^{-1} \mathrm{~s}^{-1}, 298 \mathrm{~K}$, and incident angle near the substrate normal covering the $30-1000 \mathrm{~nm}$ average film thickness $(\bar{h})$ range is investigated through scanning tunneling microscopy (STM) complemented with oxygen-adatom electrosorption measurements. The STM images of the deposits reveal a columnar structure resulting from a mechanism involving shadowing and surface diffusion. Quantitative data are obtained directly from STM images. The height distribution $N(h)$ of the interface obeys an $N(h) \propto e^{-k h}$ relationship. For $\bar{h}<500 \mathrm{~nm}$, the interface thickness $(\xi)$ increases as a power law with $\bar{h}$, whereas for $\bar{h}>500 \mathrm{~nm}$ it reaches a steady state. Under the latter condition, $\xi$ depends on the STM scan length $(S)$ as $\xi \propto S^{\alpha}$ with $\alpha$ close to $\frac{1}{3}$. These results indicate that the growth process of the gold deposits results in compact nonfractal structures with self-affine fractal surfaces, as predicted by ballistic deposition models. However, the latter fail to describe some aspects of the morphology and evolution of thin vapor-deposited gold films on this substrate.
\end{abstract}

PACS number(s): 68.70. $+\mathrm{w}, 68.55 . \mathrm{Jk}, 82.20 . \mathrm{Wt}, 81.15 .-\mathrm{z}$

\section{INTRODUCTION}

The growth of thin metal films from vapor deposition has received considerable scientific attention due to the application of these films in manufacturing electronic, magnetic, and optical devices [1]. The properties of these films are largely dependent on experimental conditions, i.e., growth rate [2], substrate temperature [2], and deposition angle [1]. Under nonequilibrium conditions, i.e., for growth rates between 20 and $400 \mathrm{~nm} \mathrm{~s}^{-1}$ and temperatures lower than $0.25 T_{m}$, where $T_{m}$ is the melting temperature, the morphology of the thin metal films can be described as a columnar structure [3]. This type of structure results from a competitive growth process, which produces shadowing between the columns, and surface diffusion of the deposited metal atoms, which tends to smooth irregularities smaller than the diffusion length $[4,5]$.

Depending on the scale range, the morphological evolution of vapor-deposited films can be followed by two different approaches. The first one is based on ballistic models $[6,7]$, i.e., particles arrive to the substrate surface following ballistic trajectories rather than random walks.
The results obtained from large-scale computer simulations with a particle incident angle normal to the substrate indicate that deposits with a nonfractal compact structure and a self-affine fractal surface are formed [8]. For large angles of incidence the columnar structure becomes more evident [9]. In both cases, the deposit surface resulting from the computer simulations exhibits holes extending down to the atomic scale. However, real coatings are smooth in the range $<10 \mathrm{~nm}$ due to surface diffusion of the deposited particles [5]. Thus, to describe the macroscopic morphological evolution of thin films, other models such as the grass or continuum models based on the Huygens principle have been developed $[10,11]$. Computer simulations of these models seem to generate columnar structures with a self-similar fractal character [5]. However, the possibility of checking the validity of the different models has been hampered by the lack of high-quality experimental data on thin-film morphology. Hitherto, perhaps, the main experimental work on this subject dealt with films at the macroscopic level [12], i.e., at a scale range far from that of the simulations. At present, scanning tunneling microscopy (STM) appears as a very suitable technique of investigating the 
structure and growth mechanism of thin metal films, as it is a nondestructive imaging technique providing threedimensional (3d) high resolution [13]. Its applicability to the study of surface roughness of thin films has been already proved $[14,15]$.

In this paper the characteristics of vapor-deposited gold films grown under nonequilibrium conditions are investigated by using STM complemented with oxygenatom electroadsorption techniques. The height distribution at the deposit surface and the interface thickness evolution with $\bar{h}$ have been obtained from the STM topography. Results are compared to the predictions of the different models for thin-film growth. It is concluded that the structure of our gold deposits is columnar with a height distribution and interface thickness evolution, in agreement with those resulting for deposits generated by ballistic deposition at near-normal-incident particle angles, although these models fail to describe other essential aspects of the thin-film morphology.

\section{EXPERIMENT}

Gold films were grown on smooth glass substrates previously cleaned in an ultrasonic bath by sequentially using water, trichloroethylene, acetone, and ethanol. The glass substrate was characterized by atomic force microscopy, which revealed a substrate corrugation less than 0.5 $\mathrm{nm}$ [16]. Gold deposits were prepared in an evaporator chamber. The angle between the direction of the incident particles and the substrate normal was set in the range between $2^{\circ}$ and $25^{\circ}$. The following experimental conditions were used at the evaporator chamber: pressure, $P=10^{-4}$ Torr; average deposit growth rate, $v=30 \mathrm{~nm} \mathrm{~s}^{-1}$; and substrate temperature, $T=298 \mathrm{~K}$. The average film thickness $\bar{h}$ of each deposit was measured with a profilometer and it was varied from 30 to $1000 \mathrm{~nm}$.

A piezo-tube STM operating in air was used to study the surface morphology of the deposits. The piezo tube was calibrated by imaging highly oriented pyrolytic graphite (HOPG) (Fig. 1). The tips were made directly by cutting $0.5-\mathrm{mm}$-diam platinum wires. To minimize

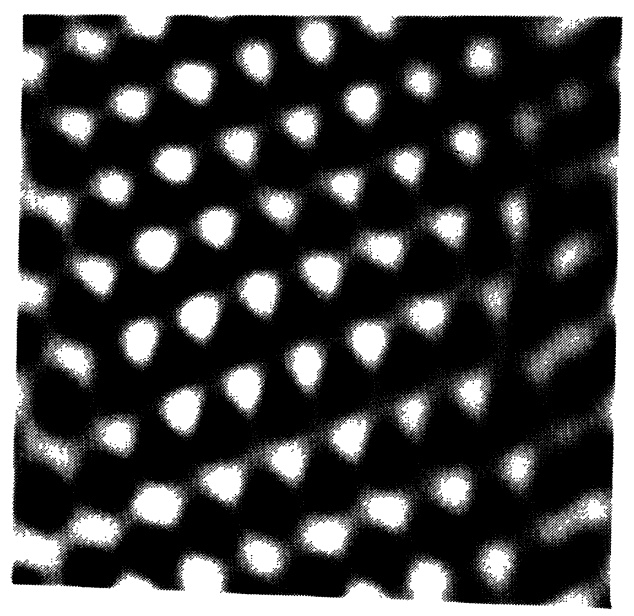

FIG. 1. $1.5 \times 1.5 \mathrm{~nm}^{2}$ top-view STM image of HOPG. occasional tip geometric artifacts different tips were used, although no influence by the tip shape was detected in our experimental data. However, a detailed study on tip effects would require a comparative analysis of STM imaging of these gold films with tips prepared by different procedures, i.e., mechanically cutting and electrochemically etching. This study will be the subject of a future paper.

STM measurements were made using a bias voltage of $0.05 \mathrm{~V}$ with the tip ( + ) at a constant current of $1-2 \mathrm{nA}$. The data were acquired in a fully automated workstation and stored as digitized images with $256 \times 256$ pixels. Occasionally, scanning electron microscopy (SEM) imaging of these films was performed.

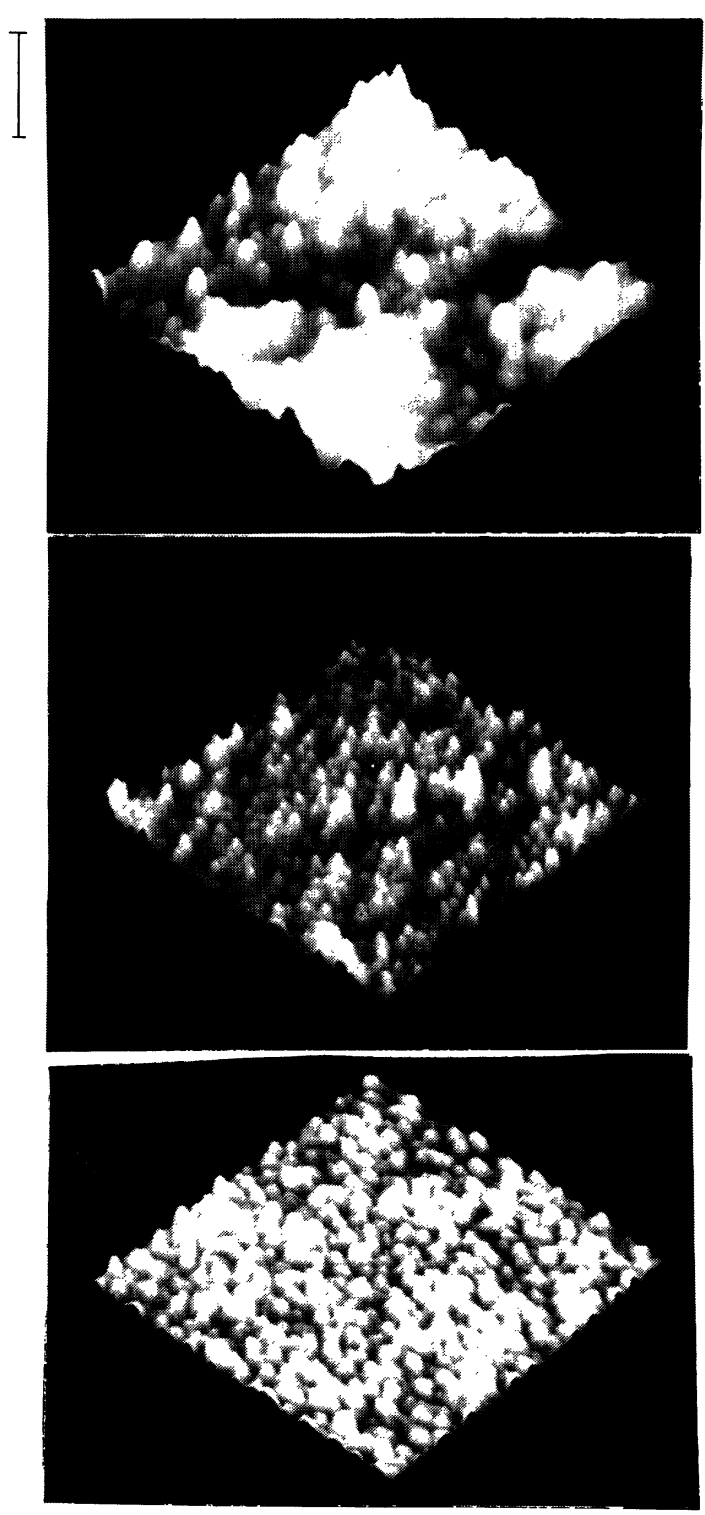

(c)

(b)

(a)

FIG. 2. 3D STM images of a vapor-deposited Au film. (a) $640 \times 640 \mathrm{~nm}^{2}, \bar{h}=30 \mathrm{~nm}$; (b) $600 \times 600 \mathrm{~nm}^{2}, \bar{h}=160 \mathrm{~nm}$; (c) $510 \times 510 \mathrm{~nm}^{2}, \bar{h}=850 \mathrm{~nm}$. The bar indicates $18 \mathrm{~nm}$ in the $z$ direction. 
The real surface area of the deposits referred to the substrate area, $A_{f}$, was determined from the oxygenadatom electrodesorption voltammetric charge in a conventional electrochemical cell containing deaerated $0.5 \mathrm{M}$ $\mathrm{H}_{2} \mathrm{SO}_{4}$ [17]. For this purpose a monolayer of oxygen adatoms was voltammetrically formed on the gold deposits and subsequently the monolayer was voltammetrically electroreduced and the electrodesorption charge $(Q)$ measured from the voltammogram [18]. The value of $A_{f}$ for each film was calculated from [17]

$$
A_{f}=\left(Q / q_{m} A_{s}\right),
$$

where $q_{m}$ is the oxygen-adatom monolayer charge density $\left(q_{m}=0.42 \mathrm{mC} \mathrm{cm}^{-2)}\right.$ and $A_{s}$ is the geometric substrate area.

\section{RESULTS}

STM images of thin gold deposits of different thickness grown at $v=30 \mathrm{~nm} \mathrm{~s}^{-1}$ and $T=298 \mathrm{~K}$ reveal a porous microstructure formed by rounded grains, which correspond to the top of columnar elements that are separated by voided regions [Fig. 2(a)-2(c)]. The column tops, which appear rather smooth and free of defects even at higher magnifications, exhibit preferred growth directions [Fig. 3(a)]. X-ray-diffraction measurements [Fig. 3 (b)] reveal a $\{111\}$ preferred orientation [18].

The voided regions between the columns exhibit branched patterns that become clearly distinguishable within the 40-3600 $\mathrm{nm}$ magnification range [Figs. 4(a) $-4(d)]$. Thus, the voided regions are completely absent when the films are imaged with a STM scan length close to the columnar top size, or when they are imaged by SEM in the micrometer range. This fact suggests that the surfaces of the gold deposits behave as fractals with an inner cutoff in the order of the column top size and an outer cutoff in the micrometer range.

Two additional relevant features of the film structure emerge from the STM images. The first one is the progressive increase in the columnar height difference, which results in surface roughening as the film becomes thicker [Fig. 2(a)-2(c)]. The second feature concerns the change in the average intercolumnar distance $d_{c}$ as $\bar{h}$ is increased. This conclusion can be derived from the inspection of typical STM scans for film thicknesses ranging from $30-850 \mathrm{~nm}$ (Fig. 5). For $\bar{h} \geq 500 \mathrm{~nm}$ large structures with $d_{c} \simeq 50-70 \mathrm{~nm}$ emerge from the small base corrugation with $d_{c} \simeq 30 \mathrm{~nm}$.

The progressive roughening of the gold films with $\bar{h}$ can be followed by measuring the root-mean-square height $\xi_{\text {STM }}$ from the STM images. This quantity provides an estimation of the mean interface width $[7,8]$. As can be seen in Fig. $6, \xi_{\mathrm{STM}}$ depends on the size of the STM image, i.e., the scan length $(S)$. Thus $\xi_{\text {STM }}$ increases with $S$ to approach an asymptotic value $\xi_{0}$. The latter quantity depends as a power law on $\bar{h}$, and for $\bar{h} \geq 500$ $\mathrm{nm}$ it approaches a constant value, indicating that the deposit surface has reached a steady state (Fig. 7). However, the tip geometry should influence to some extent (i.e., underestimating it) the $\xi_{\text {STM }}$ values so that we verify the $\bar{h}$ dependence of the deposit surface by another independent technique. For this purpose, the surface area per square centimeter of substrate $A_{f}$ of each film was calculated from Eq. (1) by measuring the electrodesorption charge of the oxygen monolayer. This method is commonly used in electrochemistry to measure real surface

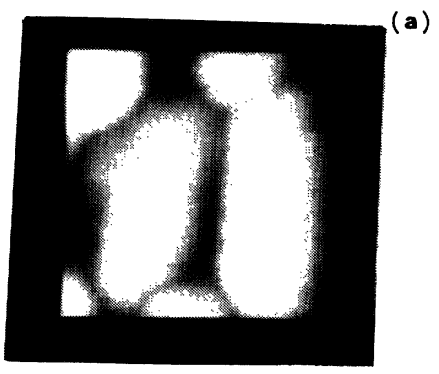


areas [17]. For $\bar{h}<500 \mathrm{~nm}$, the $A_{f}$ versus $\bar{h}$ plot (Fig. 8) shows an initial increase, whereas for $\bar{h}>500 \mathrm{~nm}$ it becomes practically independent of $\bar{h}$. This plot confirms that for $\bar{h} \geq 500 \mathrm{~nm}$ the surface of the gold deposits reaches a steady-state value. Thus we can conclude that STM actually samples the interface thickness.

Another convenient measure of the statistical properties of these surfaces is the number of points $(N)$ comprised in the $h_{m} \leq h \leq h_{M}$ range, where $h_{m}$ and $h_{M}$ are the minimum and the maximum heights at the STM image. As tip artifacts become more likely for points with low height values, the analysis of the $N(h)$ versus $h$ relationship is restricted to those points with a height greater than $0.3\left(h_{M}-h_{m}\right)$. For a given film thickness $\bar{h}$ the $\log _{10} N(h)$ versus $h$ plots yield straight lines with a slope $k$, which depends on $S$ (Fig. 9). Hence, for small $S$ values, $k$ is large and the height distribution is markedly sharp. It means that only a few columns are imaged. Otherwise, as $S$ increases and the number of imaged columns increases, the height distribution becomes broader and accordingly the value of $k$ decreases. Finally, for large $S$ values a constant $k$ is reached, indicating that an adequate statistics has been attained. For this limiting situation, the $\log _{10} N(h)$ versus $h$ plots exhibit straight lines with $k$ decreasing as $\bar{h}$ increases from 30 to $500 \mathrm{~nm}$ (Fig. 10).

\section{DISCUSSION}

The surfaces of vapor-deposited gold films resemble those described for thin films of different materials grown at deposition rates in the $20-400 \mathrm{~nm} \mathrm{~s}^{-1}$ range and $T<0.25 T_{m}[2,3]$. Under these conditions the structure of the deposits is formed by domed top columns and void domains [2].

The structure of the gold films reveals through the

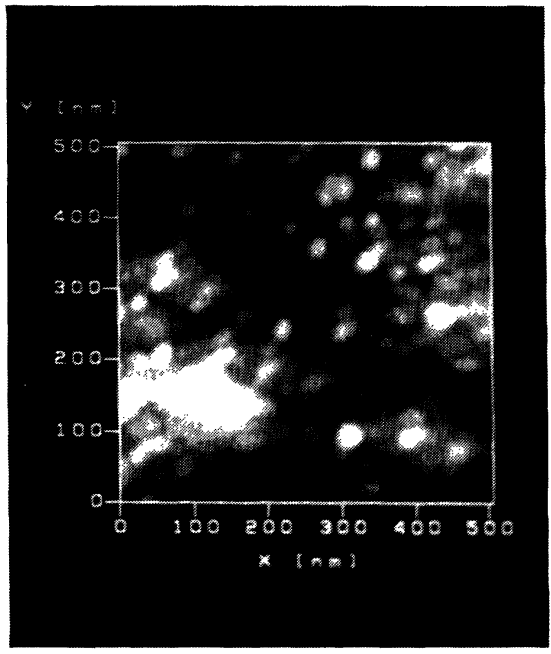

(a)

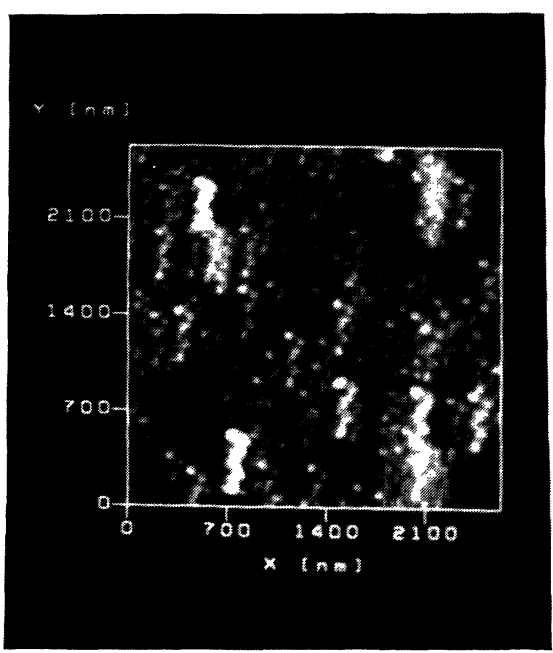

(c)

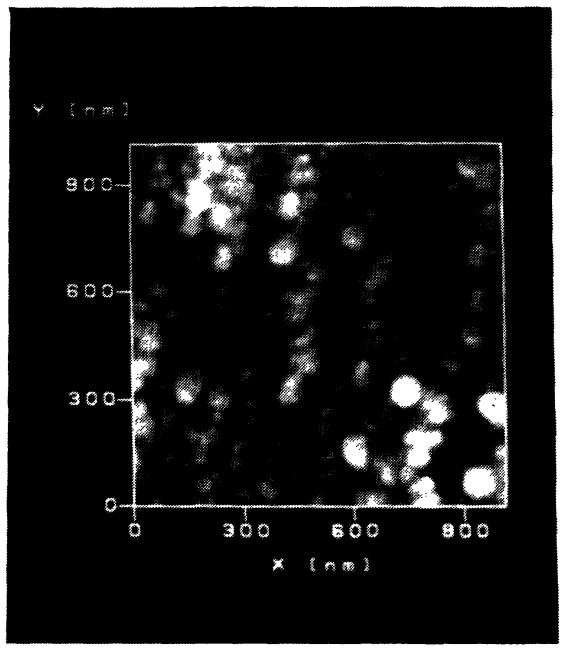

(b)

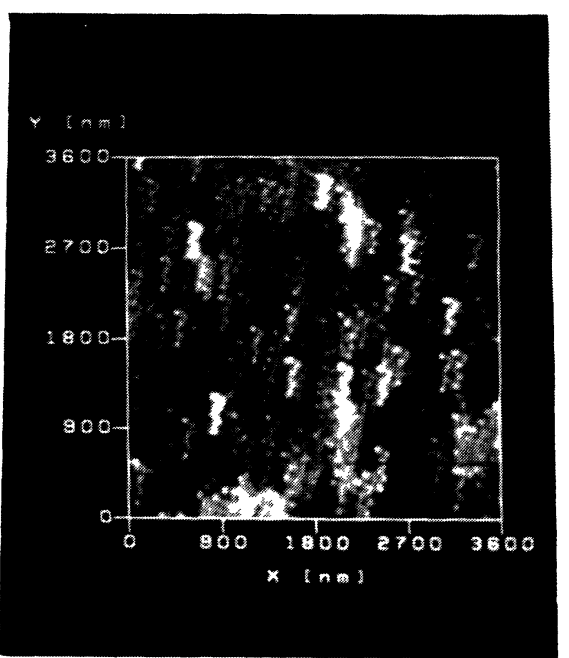

(d)

FIG. 4. Gray scale STM images (top view) of a gold deposit $(\bar{h}=850 \mathrm{~nm}$ ) at different magnifications. 


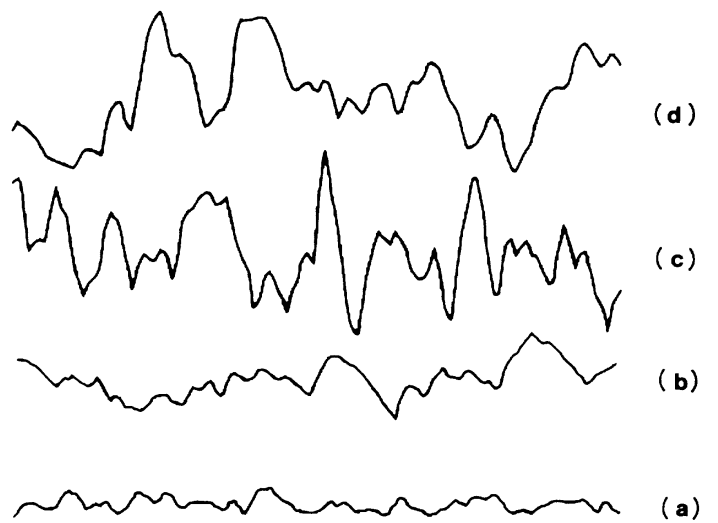

FIG. 5. Typical STM scans covering $974 \mathrm{~nm}$ for vapordeposited gold films with different $\bar{h}$ values. (a) $\bar{h}=30 \mathrm{~nm}$, (b) $\bar{h}=160 \mathrm{~nm}$, (c) $\bar{h}=500$, and (d) $\bar{h}=850 \mathrm{~nm}$. The bar indicates 6 $\mathrm{nm}$ in the $z$ direction.

STM images self-shadowing and surface diffusion as the relevant physical processes operating during growth. Self-shadowing is revealed by the progressive surface roughening with $\bar{h}$, as the winners' columns emerge from the shaded regions. Surface diffusion leads to definite
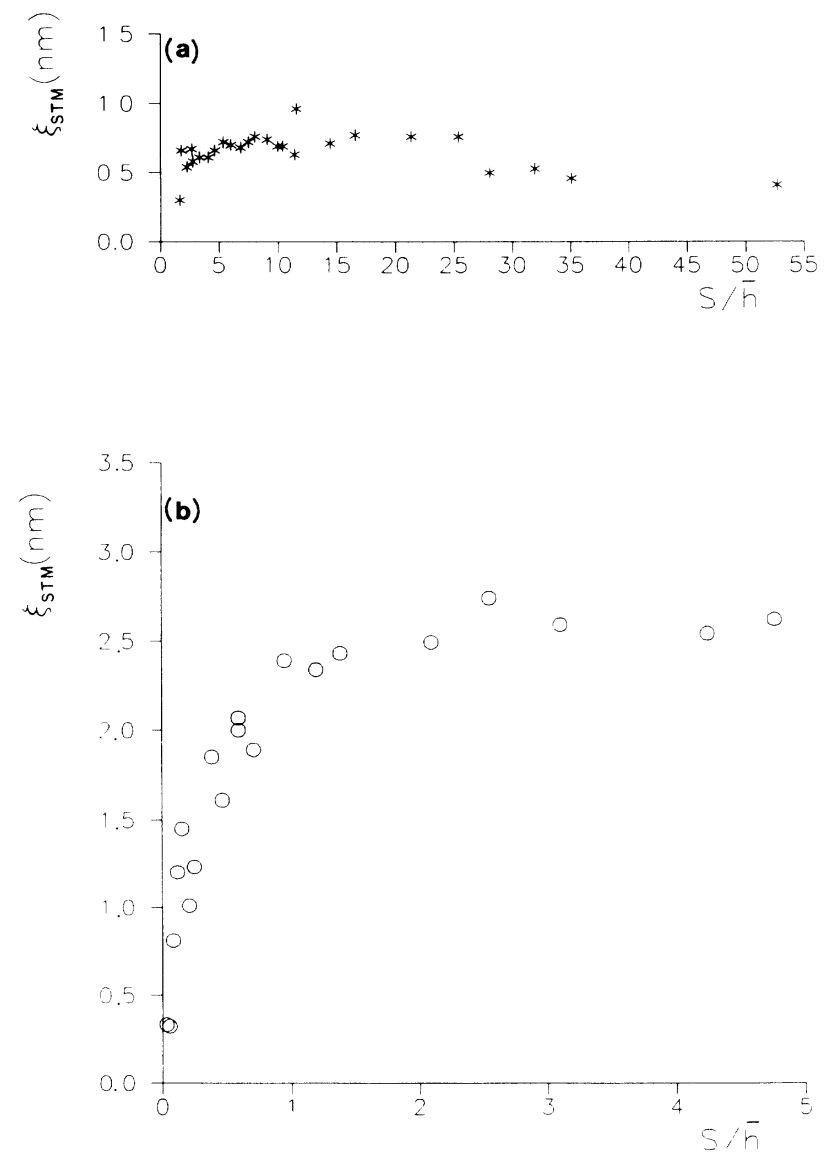

FIG. 6. $\xi_{\text {STM }}$ vs $S / \bar{h}$ plot for (a) $\bar{h}=30 \mathrm{~nm}$ and (b) $\bar{h}=850$ nm.

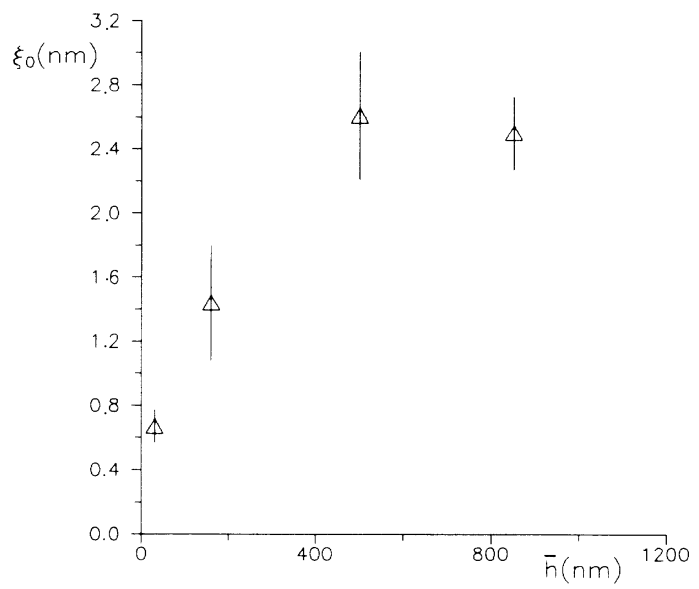

FIG. 7. $\xi_{0}$ vs $\bar{h}$ plot.

grain sizes with smooth surfaces and preferred orientation as irregularities smaller than the diffusion length of the depositing atoms are eliminated. Another important feature from the STM images is the absence of large fluctuations in height, suggesting that the surface cannot be described as a self-similar fractal. This fact can be proved quantitatively from the analysis of the height size distribution. For a self-similar fractal surface, the following equation is obeyed $[5,19]$ :

$$
N(h)=A^{\prime} h^{-\tau},
$$

where $A^{\prime}$ and $\tau$ are constants with $1<\tau<2$.

On the other hand, the linear $\log _{10} N(h)$ versus $h$ plots (Fig. 10) obtained for our deposits indicate that the height size distribution obeys the equation

$$
N(h)=A e^{-k h},
$$

where $A$ and $k$ are constants with $k$ decreasing as $\bar{h}$ increases. This type of height size distribution function was also found for self-affine fractal surfaces grown from

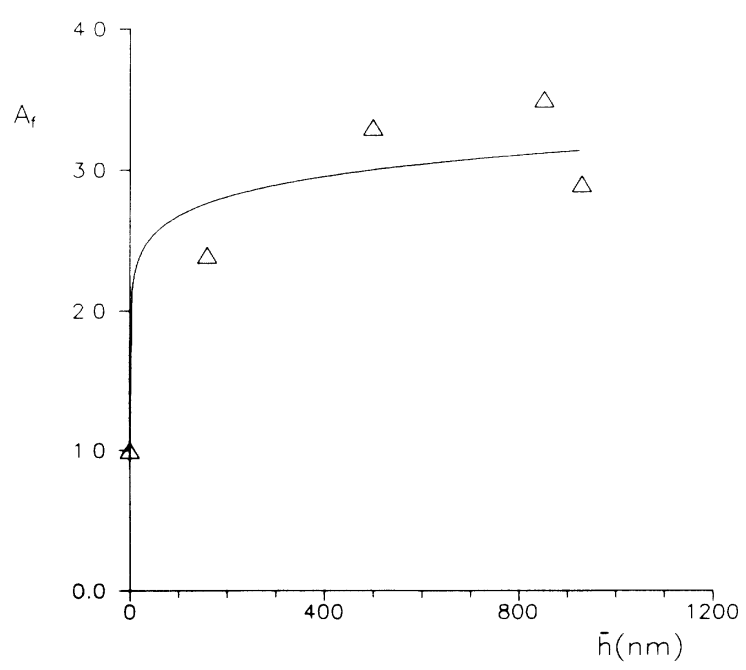

FIG. 8. $A_{f}$ vs $\bar{h}$ plot. 


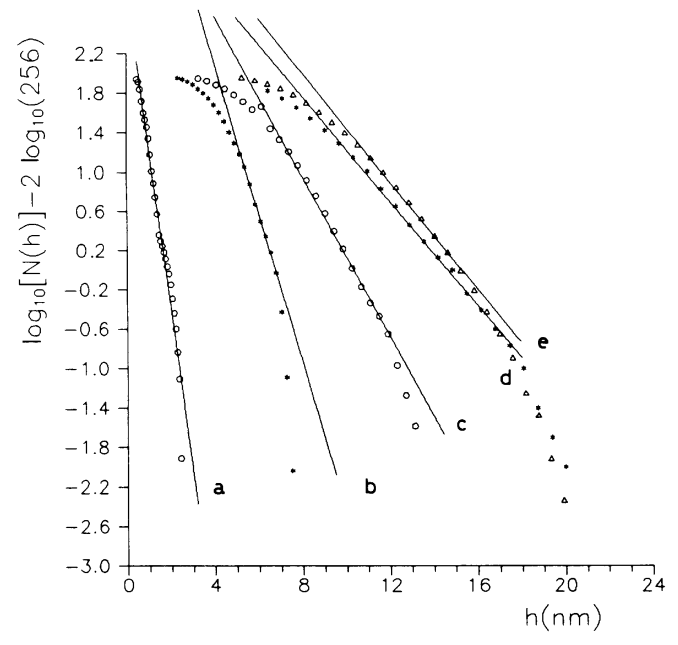

FIG. 9. $\log _{10} N(h)$ vs $h$ plots for $\bar{h}=850 \mathrm{~nm}$, (a) $S=46.7 \mathrm{~nm}$, (b) $S=214 \mathrm{~nm}$, (c) $S=605 \mathrm{~nm}$, (d) $S=1000 \mathrm{~nm}$ and (e) $S=3600$ nm.

large-scale computer simulations of ballistic models at near-normal-incident angles [8]. As this is precisely our case, it can be concluded that the surfaces of the vapordeposited gold films can be described as self-affine, rather than self-similar fractal surfaces. To characterize these self-affine fractal surfaces, the dynamic approach has been proved to be an effective tool [20]. A useful measure of the surface roughness is the interface width $\xi$ defined for a sample of lateral dimension $L[1,7,20]$. For a columnar structure $\xi$ is the mean-square average given by

$$
\xi(L) \equiv\left(\frac{1}{L} \Sigma\left[h\left(x_{i}\right)-\bar{h}\right]^{2}\right)^{1 / 2},
$$

where $\xi$ and $L$ are related through the equation

$$
\xi \cong L^{\alpha} f\left(\bar{h} / L^{\gamma}\right)
$$

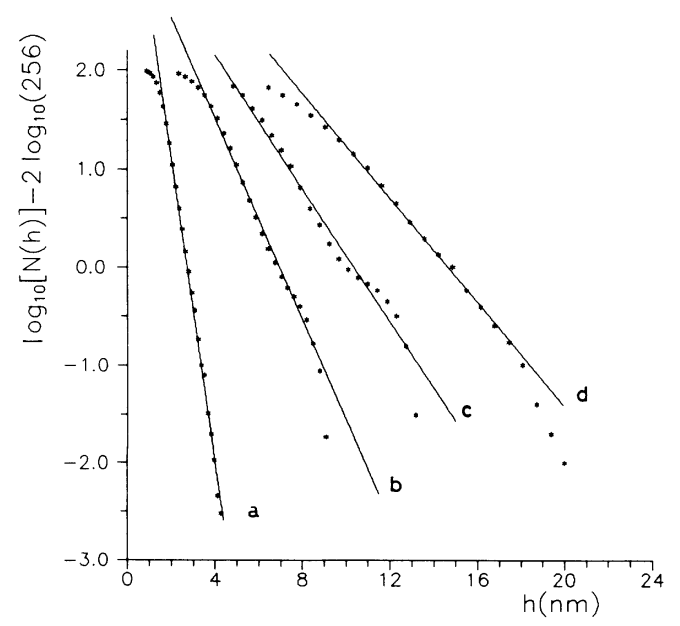

FIG. 10. $\log _{10} N(h)$ vs $h$ plots for $S=1000 \mathrm{~nm}$ (a) $\bar{h}=30 \mathrm{~nm}$, (b) $\bar{h}=160 \mathrm{~nm}$, (c) $\bar{h}=500 \mathrm{~nm}$ and (d) $\bar{h}=850 \mathrm{~nm}$. where the function $f(x)$ has the following properties: $f(x)=$ const for $x \rightarrow \infty$ and $f(x)=x^{\alpha / \gamma}$ for $x \rightarrow 0$. Thus, for $\bar{h} \gg L^{\gamma}$, Eq. (5) becomes

$$
\xi(L) \propto L^{\alpha},
$$

whereas for $\bar{h}<L$, it results in

$$
\xi(\bar{h}) \propto \bar{h}^{\beta},
$$

where $\beta$ is the kinetic roughening exponent $(\beta=\alpha / \gamma)$. The quantity $\xi$ is directly related to the $\xi_{\text {STM }}$ so that we can compare the predictions of the dynamics scaling approach to our STM data.

The exponent $\beta$ describes the growth of the surface correlations with time $t$ (or height) along the growth direction. However, it was shown from large-scale computer simulations that for $\bar{h}$ larger than a characteristic value $\bar{h}_{c}$ the surface reaches a steady state [20]. This is precisely the behavior observed for our deposits (Figs. 7,8). Despite the fact that we cannot estimate accurately the value of $\beta$ from our data, $\bar{h}_{c}$ seems to be close to 500 nm. It should be noted that for $\bar{h}>\bar{h}_{c}$, i.e., when the deposit surface has reached the steady state, the exponent $\alpha$ becomes the fundamental parameter for describing the roughness of a self-affine fractal interface [20]. Under this condition, it is known that the roughness is a horizontal-length scale-dependent variable [20, 21]. For our system this implies that, for sample thickness larger than $\bar{h}_{c}, \xi_{\text {STM }}$ and $S$ can be related by

$$
\xi_{\mathrm{STM}} \propto S^{\alpha} \text {. }
$$

The $\log _{10} \xi_{\text {sTM }}$ versus $\log _{10} S$ plot for data shown in Fig. 6(b) $\left(\bar{h}>\bar{h}_{c}\right)$ is shown in Fig. 11. Although there is a significant scatter, for $S>d_{c}$ the data collapse well onto a single line with a slope $\alpha=0.34 \pm 0.035$. This value can be directly compared to those obtained from the perime$\operatorname{ter}(P)$ /area $(A)$ analysis of the lakes or islands generated by the intersection of a plane with the deposit surface imaged by STM [22]. In this method, the fractal dimension of the lakes or islands perimeter $D^{\prime}$ and $\alpha$ are related by [7]

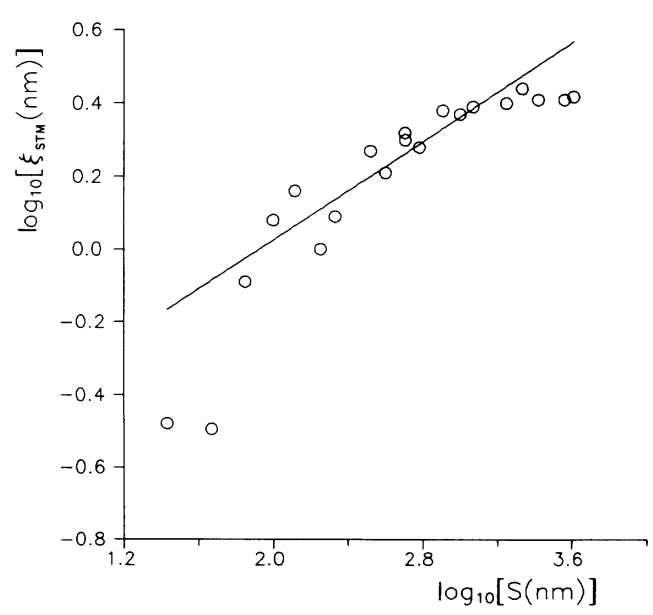

FIG. 11. $\log _{10} \xi_{\text {STM }}$ vs $S$ plot from data shown in Fig. 6(b). 


$$
D^{\prime}=d-1-\alpha,
$$

where $d$ is the space dimension where the deposit is grown. Thus the $\alpha$ values for these gold deposits can be calculated from the $D^{\prime}$ values for $A$ greater than the columnar size, which introduces a lower cutoff length [23]. The $\alpha$ versus $\bar{h}$ plot (Fig. 12) shows that $\alpha$ increases slightly with $\bar{h}$ and for $\bar{h}>500 \mathrm{~nm}$ it reaches an asymptotic value close to $\frac{1}{3}$. Thus, both methods give $\alpha \cong \frac{1}{3}$ a value that agrees very well with that expected for a selfaffine surface generated by ballistic deposition models at near-normal-incident angles [8,24,25].

Up to now very few experimental results on self-affine fractal interfaces have been published. Recently, $\alpha$ values were reported for interfaces grown from immiscible displacement in porous media [21]. Here we present experimental quantitative data on self-affine fractal properties of vapor-deposited metal films.

In principle there is a good correlation between the quantitative data derived from STM and those obtained from computer simulations using ballistic models, although the latter fail to describe other essential features of these systems. Thus, for ballistic models without restructuring, surface irregularities are present even at the atomic scale [8], whereas in the real systems the irregularities disappear at scales smaller than the columnar size. If restructuring is allowed in the simulations, then the value of $\alpha$ would increase [26] and the agreement between experimental and simulation data would vanish. In fact, we find that the ballistic models only explain the experimental data, providing that each deposited atom behaves itself as a growing column. Conversely, continuum models such as the grass model and those based on the Huygens principle account for the macroscopic evolution of the deposits by better considering the role of surface diffusion, but they seem to generate self-similar [5] rather than self-affine surfaces. These models predict

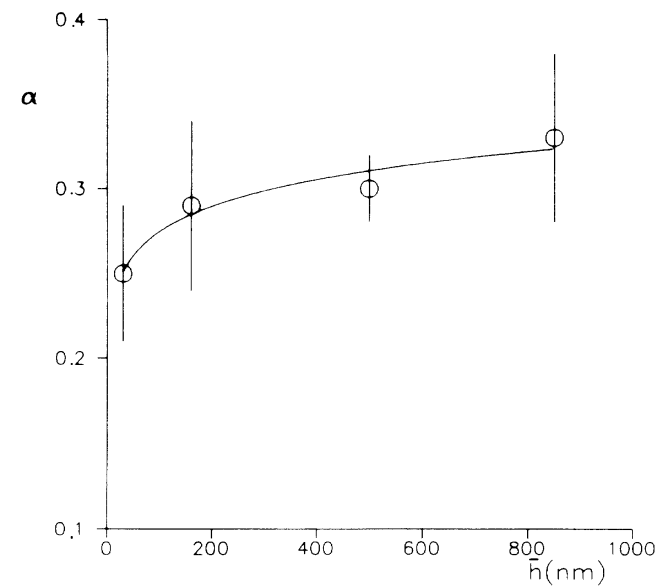

FIG. 12. $\alpha$ vs $\bar{h}$ plot.

large surface fluctuations that are not detected in our STM images.

Therefore, one can conclude that none of the present models describes completely the morphological evolution of thin gold films grown from the vapor far from the equilibrium conditions. It is evident from these results that a greater link between experimental data and theory is required. The present results also show that STM is a suitable technique of testing the validity of different models on thin-film morphology and growth.

\section{ACKNOWLEDGMENTS}

We are indebted to J. Gómez-Rodríguez for fruitful discussions. We also thank J. Herrero from the CIEMAT for technical assistance in the gold film depositions. This work was partially supported by CICYT (Spain) under Project No. MAT89-0204.
[1] P. Meakin, Crit. Rev. Solid State Mater. Sci. 13, 147 (1987).

[2] B. Movchan and A. V. Demchishin, Phys. Met. Metallogr. (USSR) 28, 83 (1969).

[3] D. J. Srolovitz, A. Mazor, and B. G. Bukiet, J. Vac. Sci. Technol. A 6, 2371 (1988).

[4] Thin Film Process, edited by J. Vossen and W. Kern (Academic, New York, 1978), Pt. 2.

[5] G. S. Bales, R. Bruinsma, E. A. Eklund, R. P. U. Karunasiri, J. Rudnick, and A. Zangwill, Science 249, 264 (1990).

[6] J. M. Vold, J. Colloid Sci. 14, 168 (1959).

[7] T. Vicsek, Fractal Growth Phenomena (World Scientific, Singapore, 1989).

[8] P. Meakin, P. Ramanlal, L. M. Sander, and R. C. Ball, Phys. Rev. A 34, 509 (1986).

[9] J. Krug and P. Meakin, Phys. Rev. A 43, 900 (1991).

[10] R. P. U. Karunasiri, R. Bruinsma, and J. Rudnick, Phys. Rev. Lett. 62, 788 (1989).
[11] C. Tang, S. Alexander, and R. Bruisma, Phys. Rev. Lett. 64, 772 (1990).

[12] R. Messier and J. E. Yehoda, J. Appl. Phys. 58, 3739 (1986).

[13] P. K. Hansma and J. Tersoff, J. Appl. Phys. 61, R1 (1987).

[14] C. Schönenberg, S. F. Alvarado, and C. Ortiz, J. Appl. Phys. 66, 4258 (1989).

[15] E. A. Eklund, R. Bruinsma, J. Rudnick, and R. S. Williams, Phys. Rev. Lett. 67, 1759 (1991).

[16] W. A. Huang (private communication).

[17] S. Trassati and O. Petrii, Pure Appl. Chem. 67, 711 (1991).

[18] S. Manne, J. Massie, B. Elings, P. K. Hansma, and A. A. Gerwirth, J. Vac. Sci. Technol. B 9, 950 (1991).

[19] B. B. Mandelbrot, The Fractal Geometry of Nature (Freeman, New York, 1982).

[20] F. Family, Physica A 168, 561 (1990).

[21] M. A. Rubio, C. A. Edwards, A. Dougherty, and J. P. Gallub, Phys. Rev. Lett. 63, 1685 (1989). 
[22] J. Gómez-Rodríguez, A. M. Baró, L. Vázquez, R. C. Salvarezza, J. M. Vara, and A. J. Arvia, J. Phys. Chem. 96, 347 (1992).

[23] P. Ocón, P. Herrasti, L. Vázquez, R. C. Salvarezza, J. M. Vara, and A. J. Arvia, Electrochim. Acta (to be pub- lished).

[24] P. Meakin and J. Krug, Europhys. Lett. 11, 7 (1990).

[25] J. Kim and J. Kosterlitz, Phys. Rev. Lett. 62, 2289 (1989).

[26] P. Meakin and R. Jullien, Phys. Rev. A 41, 983 (1990). 


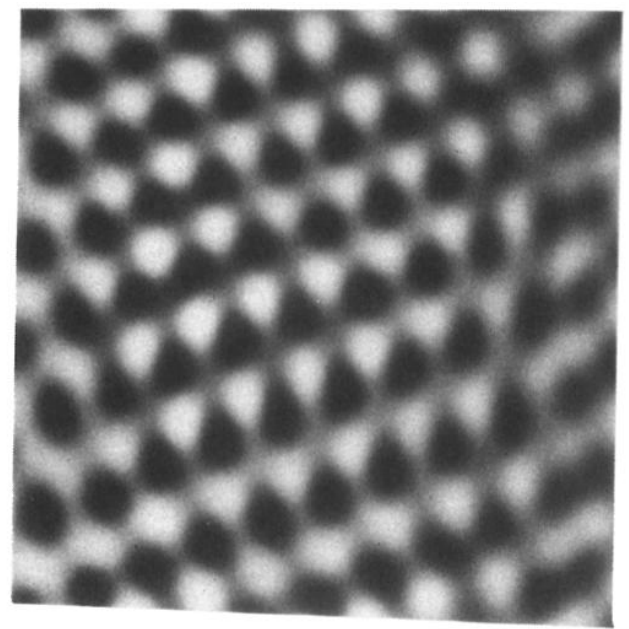

FIG. 1. $1.5 \times 1.5 \mathrm{~nm}^{2}$ top-view STM image of HOPG. 


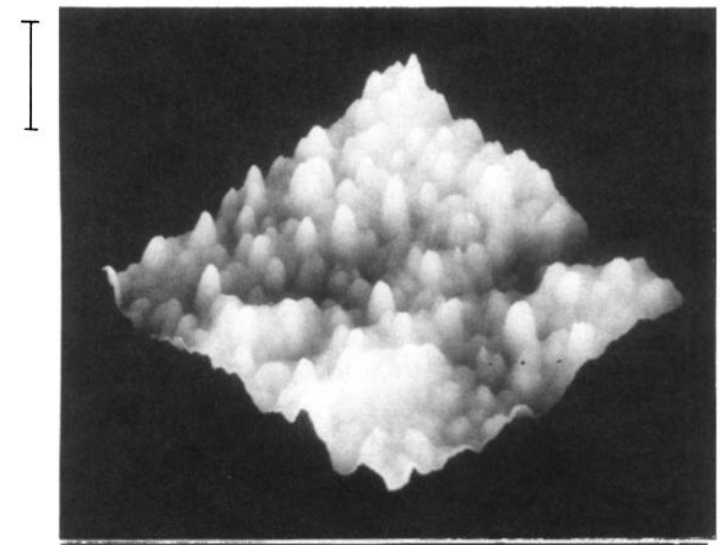

(c)

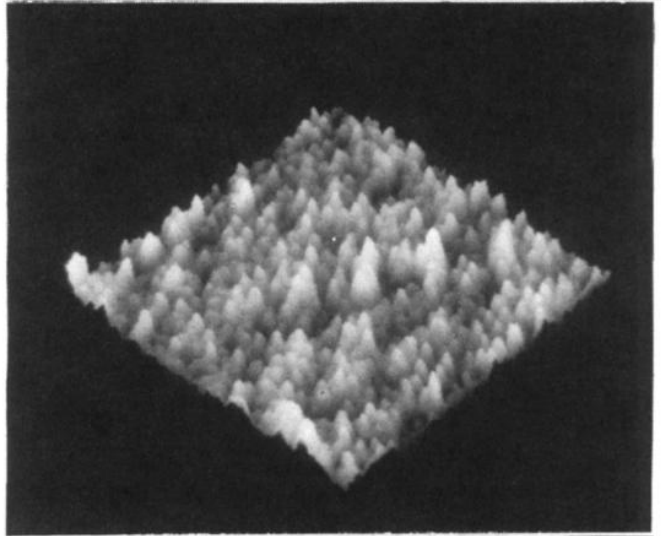

(b)

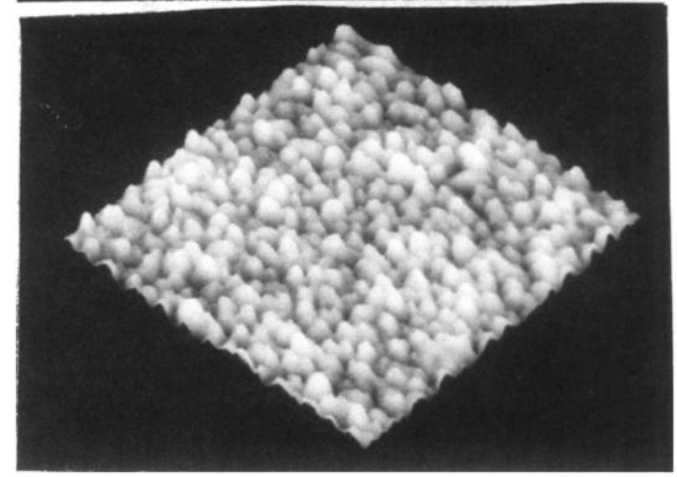

(a)

FIG. 2. 3D STM images of a vapor-deposited Au film. (a) $640 \times 640 \mathrm{~nm}^{2}, \bar{h}=30 \mathrm{~nm}$; (b) $600 \times 600 \mathrm{~nm}^{2}, \bar{h}=160 \mathrm{~nm}$; (c) $510 \times 510 \mathrm{~nm}^{2}, \bar{h}=850 \mathrm{~nm}$. The bar indicates $18 \mathrm{~nm}$ in the $z$ direction. 


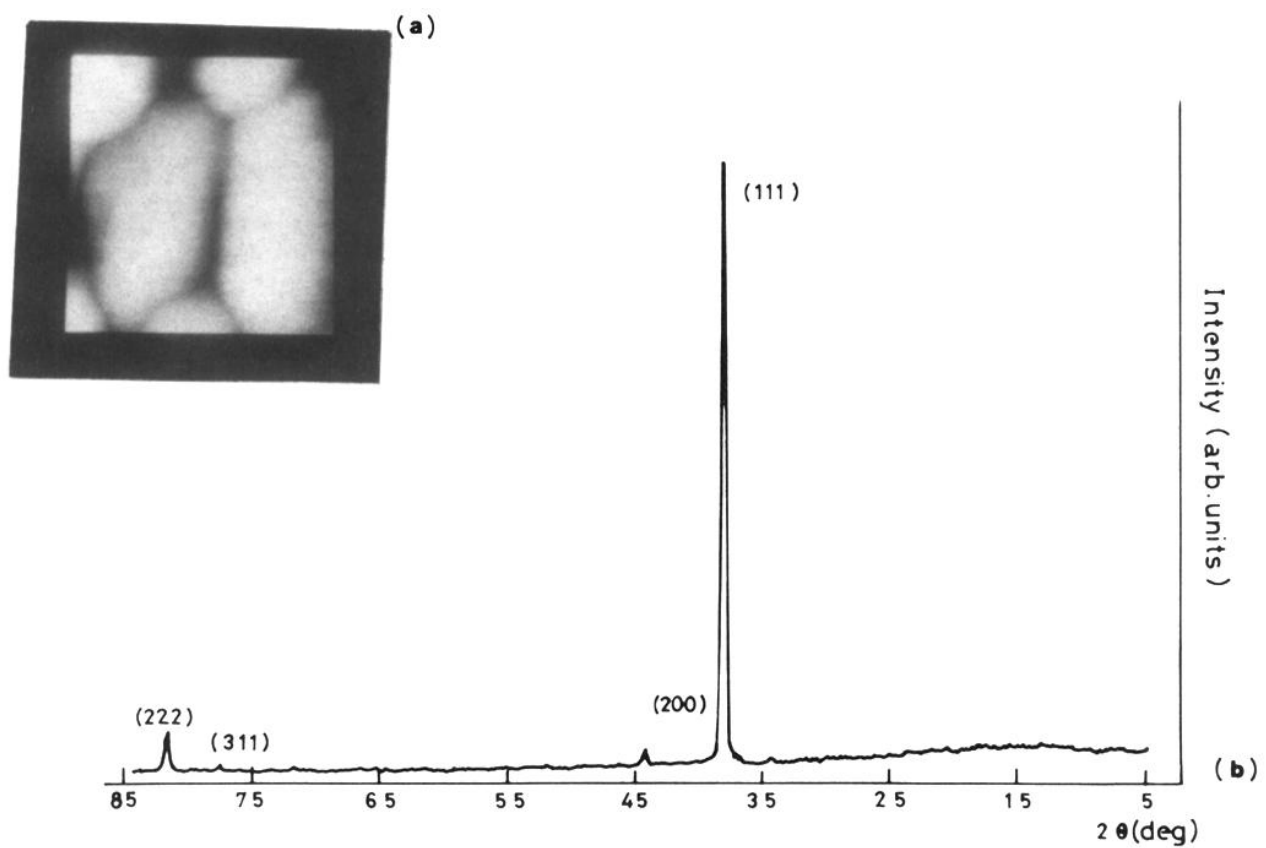

FIG. 3. (a) $70 \times 70 \mathrm{~nm}^{2}$ gray scale STM image (top view) of a gold deposit ( $\bar{h}=160 \mathrm{~nm}$ ). (b) X-ray-diffraction pattern corresponding to a vapor gold film. 


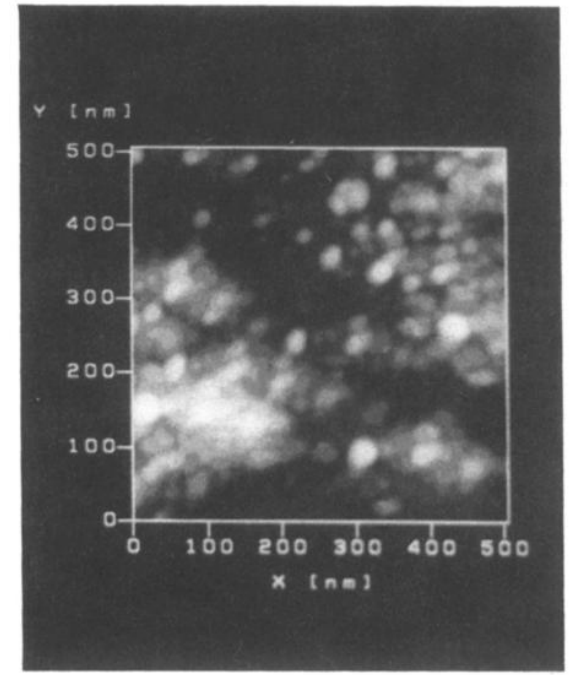

(a)

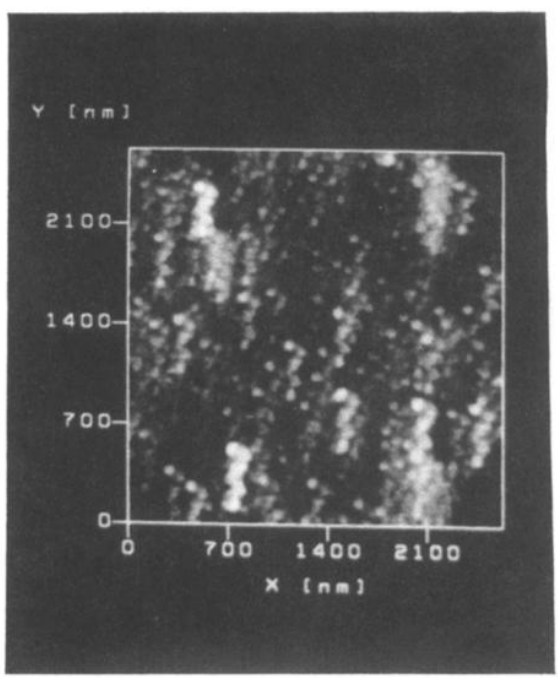

(c)

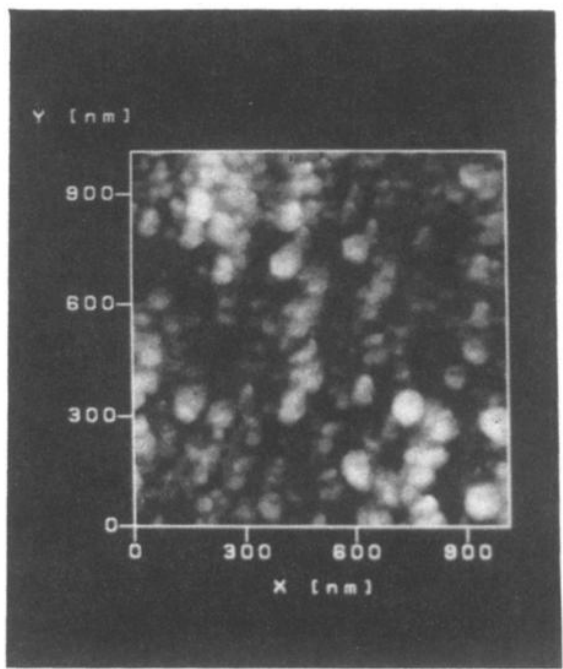

(b)

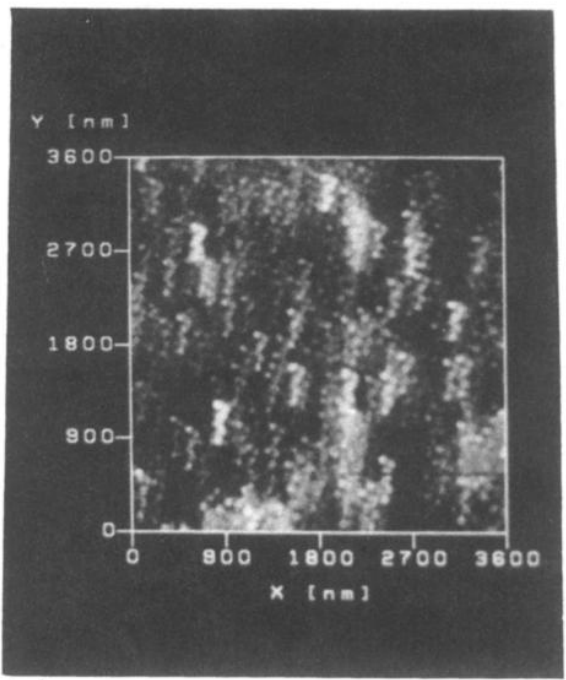

(d)

FIG. 4. Gray scale STM images (top view) of a gold deposit $(\bar{h}=850 \mathrm{~nm}$ ) at different magnifications. 